\title{
Giant Tumor of the perineal region.
}

\author{
Ines Lahouel ${ }^{1}$, Nesrine Ben Salah ${ }^{1}$, Wadii Hamdouni ${ }^{1}$, Hichem Belhadjali ${ }^{1}$, and \\ Jameleddine Zili ${ }^{1}$ \\ ${ }^{1}$ Fattouma Bourguiba University Hospital of Monastir
}

November 30, 2021

\section{Giant Tumor of the perineal region}

Key clinical message: Giant condyloma acuminatum, also known as, Buschke-Lowenstein tumour is a very rare, sexually transmitted disease. It is a slow-growing, destructive lesion that affects the anogenital region. The HPV has been identified as an important contributory factor in the development of this tumor.

Key words: Dermatology, oncology, Giant condyloma acuminatum, HPV.

A 48-year old man presented with a giant lesion of the perineal region which appeared 10 years before and gradually increased in size. Clinical examination revealed a lobulated, exophytic, non-painful, non-bleeding, cauliflower-shaped lesion on the penis and the scrotum, measuring approximately $8 \times 7 \mathrm{~cm}$ suggestive of a giant condyloma acuminatum (Figure 1). There were no enlarged inguinal nodes. Magnetic resonance imaging of the pelvis and the abdomen revealed no regional lymph node involvement or evidence of distant metastasis. A skin biopsy confirmed the diagnosis of papillary condyloma acuminatum secondary to human papillomavirus (HPV). Radical excision of the entire lesion showed no signs of invasion. Blood tests for detecting Human Immunodeficiency Virus (HIV), Hepatitis B Virus (HBV), Hepatitis C virus (HCV) and syphilis were negative. Giant condyloma acuminatum, also known as, Buschke-Lowenstein tumour is a very rare, sexually transmitted disease. ${ }^{1,2}$ It is a slow-growing, expansive, cauliflower-like, destructive lesion that affects the anogenital region. ${ }^{1,2}$ The HPV has been identified as an important contributory factor in the development of this tumor. ${ }^{1,2}$

\section{Authors' contributions:}

Ines Lahouel: Conceptualization; data curation; formal analysis; investigation; methodology; resources; validation; visualization; writingoriginal draft; writing-review and editing.

Nesrine Ben Salah: Conceptualization; data curation; formal analysis; investigation; methodology; resources; validation; visualization; writingoriginal draft; writing-review and editing.

Wadii Hamdouni: Investigation; methodology.

Hichem Belhadjali: Investigation; methodology.

Jameleddine Zili: Investigation; methodology; project administration; resources; supervision; validation; visualization; writing-original draft.

\section{References:}

Uth Ovesen A. Perianal Buschke-Löwensteins tumor [Perianal Buschke-Löwenstein tumour]. Ugeskr Laeger . 2012 Jun 4;174(23):1616-7.

Papiu HS, Dumnici A, Olariu T, et al. Perianal giant condyloma acuminatum (Buschke-Löwenstein tumor). Case report and review of the literature. Chirurgia (Bucur) . 2011 Jul-Aug;106(4):535-9. 
Data sharing not applicable-no new data generated

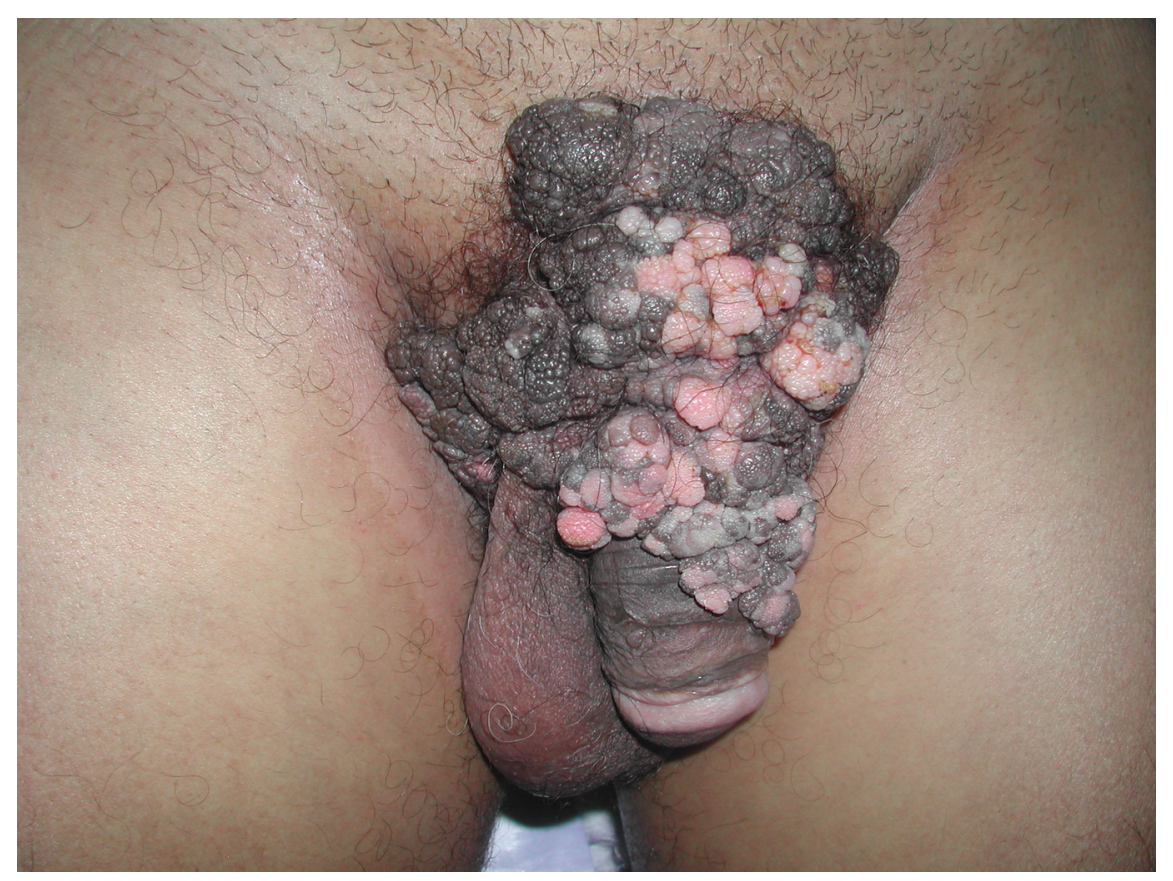

